\title{
GMR
}

\section{Expression pattern of JMJD1C in oocytes and its impact on early embryonic development}

\author{
C.H. Li ${ }^{1,2 *}$, Y. Gao ${ }^{1 *}$, S. Wang ${ }^{1}$, F.F. Xu' ${ }^{1}$, L.S. Dai ${ }^{1}$, H. Jiang ${ }^{1}$, X.F. Yu ${ }^{1}$, C.Z. Chen ${ }^{1}$, \\ B. Yuan ${ }^{1}$ and J.B. Zhang ${ }^{1,3}$ \\ ${ }^{1}$ Laboratory Animal Center, College of Animal Sciences, Jilin University, \\ Changchun, Jilin, China \\ ${ }^{2}$ College of Life Sciences, Baicheng Normal University, Baicheng, Jilin, China \\ ${ }^{3}$ Jilin Provincial Key Laboratory of Animal Embryo Engineering, \\ College of Veterinary Medicine, Jilin University, Changchun, Jilin, China \\ *These authors contributed equally to this study. \\ Corresponding authors: B. Yuan / J.B. Zhang \\ E-mail: yuanbao1982@163.com / zhangjiabaojlu@163.com
}

Genet. Mol. Res. 14 (4): 18249-18258 (2015)

Received August 12, 2015

Accepted October 25, 2015

Published December 23, 2015

DOI http://dx.doi.org/10.4238/2015.December.23.12

\begin{abstract}
Cell reprogramming mediated by histone methylation and demethylation is crucial for the activation of the embryonic genome in early embryonic development. In this study, we employed quantitative realtime polymerase chain reaction (qRT-PCR) to detect mRNA levels and expression patterns of all known histone demethylases in early germinal vesicle stage and in vitro-matured metaphase II (MII) oocytes (which are commonly used as donor cells for nuclear transfer). On screening, the Jumonji domain containing 1C (JMJD1C) gene had the highest level of expression and hence was used for subsequent experiments. We also found that JMJD1C was primarily expressed in the nucleus and showed relatively high levels of expression at the 2-cell, 4-cell, 8-cell, 16-cell, morula, and blastocyst stages of embryos developed from MII oocytes fertilized in vitro. Further, we knocked down the JMJD1C gene in MII oocytes using siRNA
\end{abstract}


and monitored the cleavage of zygotes and development of early embryos after in vitro fertilization. The results showed that the zygote cleavage and blastocyst rates of the transfection group were reduced by $57.1 \pm 0.07$ and 50 $\pm 0.01 \%$ respectively, which were significantly lower than those of the negative control group $(\mathrm{P}<0.05)$. These data suggest that JMJD1C plays a key role in the normal development of early bovine embryos. Our results also provide a theoretical basis for the investigation of the role and molecular mechanism of histone demethylation in the early development of bovine embryos.

Key words: JMJD1C; Demethylase; Oocyte; Early embryo; Expression pattern

\section{INTRODUCTION}

In germ cells and early embryos, the entire genome is dynamic due to genome-wide reprogramming. The discovery of histone demethylase (HDM) demonstrated that methylation is a reversible process and opened a new research area for detailed investigation of histone modifications. Recent research shows that the level of H3K9 methylation is crucial for pluripotent stem cells to stay in an intermediate state as evidenced by its role in full reprogramming of somatic cells to induced pluripotent stem cells (Chen et al., 2013). HDM proteins can be classified further into subfamilies on the basis of sequence homology and members of a subfamily can only perform the demethylation on the same lysine residues.

JMJD1C gene is one of the three members in the JMJD1 family. JMJD1C can specifically prevent H3K9me1 and H3K9me2 methylation, and has no catalytic effect on H3K9me3 (Okada et al., 2007; Kim et al., 2010; Kim et al., 2012). Studies have shown that the promoter region of JMJD1C gene has POU5F1/OCT-4 binding sites. Since POU5F1/OCT-4 is the key transcription factor for stem cells to maintain pluripotency, POU5F1/OCT-4-mediated JMJD1C transcription might play an important role in the regulation of gene expression in undifferentiated stem cells (Chen et al., 2013). However, the function of JMJD1C in early bovine embryonic development remains unclear.

To further elucidate the role of HDM proteins during early embryonic development in bovines and to screen for marker HDM proteins that play important roles in this process, we have 1) analyzed mRNA levels and expression patterns of HDM family of genes at the GV stage and in MII bovine oocytes, 2) identified JMJD1C as the gene with highest relative expression in bovine oocytes, 3) detected the expression pattern of JMJD1C during early bovine embryonic development after IVF, 4) knocked down JMJD1C in oocytes using siRNA microinjection, and 5) measured zygote cleavage and early embryonic development after IVF. We have also presented the expression patterns of bovine HDM family members in oocytes and discussed the functions of JMJD1C in early embryonic development. Our analysis provides a theoretical basis for improving the procedures that are involved in somatic cell nuclear transfer.

\section{MATERIAL AND METHODS}

\section{Materials}

An oocyte maturation medium was prepared by mixing M199 medium (Gibco, Life technologies, Grand Island, NY, USA) with 10\% (v/v) FBS (Hyclone, Logan, UT, USA), 1\% Pyruvate 
(Sigma-Aldrich, Shanghai, China), 0.5\% FSH (Sigma-Aldrich, Shanghai, China), 0.1\% $\beta$-estradiol (Sigma-Aldrich, Shanghai, China) and 1\% penicillin/streptomycin (PAA, Cambridge, England). The medium was divided into $100 \mu \mathrm{L}$ droplets, overlaid with mineral oil and incubated at $39^{\circ} \mathrm{C}$ and $5 \%$ $\mathrm{CO}_{2}$ to reach equilibrium.

\section{Preparation of GV and MII bovine oocytes}

Fresh bovine ovaries were acquired from slaughterhouses and washed with preheated saline at $39^{\circ} \mathrm{C}$ containing penicillin and streptomycin. A syringe with a 20-gauge needle was used to aspirate the follicular fluid from follicles that were $3-8 \mathrm{~mm}$ in diameter. The cumulus-oocyte complexes (COCs) were selected under a stereoscopic microscope. Some of the COCs were treated with $0.2 \%$ hyaluronidase in an incubator for $1 \mathrm{~min}$, followed by vortexing to remove cumulus cells. These GV oocytes were collected and placed in liquid nitrogen.

The remaining COCs were transferred to droplets of the maturation medium and incubated at $39^{\circ} \mathrm{C}$ and $5 \% \mathrm{CO}_{2}$ for $22 \mathrm{~h}$. The COCs that had matured in vitro were retrieved and the cumulus cells were removed using hyaluronidase. MII oocytes in which the first polar body had discharged were selected under a microscope, washed, collected and placed in liquid nitrogen.

\section{RNA extraction and gene expression analysis}

RNeasy Micro Kit (QIAGEN, Hilden, Germany) was used to extract total RNA. The number of GV and MIl oocytes used and the RNA concentrations are presented in Table 1.

Table1. Total RNA yield from bovine oocytes.
\begin{tabular}{lccc}
\hline Stage & Number of Oocytes & Quantity $(\mathrm{ng} / \mathrm{\mu l})$ & $\mathrm{A}_{2601280}$ \\
\hline GV & 180 & 60 & 1.88 \\
MII & 180 & 71 & 1.81 \\
\hline
\end{tabular}

PrimeScript RT reagent kit (TaKaRa, Dalian, China) was used for reverse transcription to obtain cDNAs of GV and MII oocytes. SYBR Green Real-time PCR Master Mix (TaKaRa) dye and MasterCycler RealPlex (Eppendorf, Hamburg, Germany) were used to detect the relative levels of the expression of genes related to histone demethylation at GV and MII stages. The primer sequences and length of amplified fragments of individual genes are summarized in Table 2 . The formula $2^{-\Delta \Lambda C t}$ was used to calculate the relative mRNA expression levels of JMJD1C and the HDM family genes in both oocytes and embryos treated with different siRNAs.

\section{In vitro fertilization of bovine oocytes}

Frozen semen was thawed in a $37^{\circ} \mathrm{C}$ water bath for $30 \mathrm{~s}$ and poured into $10 \mathrm{~mL}$ Dulbecco's phosphate-buffered saline (D-PBS) containing $10 \%$ penicillin/streptomycin at $37^{\circ} \mathrm{C}$. After gentle mixing and centrifugation, cells were resuspended in $1 \mathrm{~mL} \mathrm{D-PBS}$ with $10 \%$ penicillin/streptomycin. Following another centrifugation, the cells were resuspended in 1-2 $\mathrm{mL}$ in vitro fertilization (IVF) stock solution (Table 3) containing $60 \mu \mathrm{g} / \mathrm{mL}$ heparin. Sperm density was determined under a 
microscope to be approximately $1 \times 10^{6} / \mathrm{mL}$. Fertilization droplets were then produced.

Mature COCs were washed with D-PBS and IVF stock solution before being transferred to the IVF droplets. Oocytes were removed after 24-26 h, washed in D-PBS, shaken for $1.5 \mathrm{~min}$ to remove cumulus cells, and transferred to in vitro culture (IVC) droplets at a concentration of 10-15 cells per droplet. The composition of IVC medium is provided in Table 4.

Table 2. Sequence of qRT-PCR primers used in this study.

\begin{tabular}{|c|c|c|}
\hline Gene & Primer sequence $\left(5^{\prime}-3^{\prime}\right)$ & PCR fagment length (bp) \\
\hline \multirow[t]{2}{*}{ GAPDH } & F-GCCATCAATGACCCCTTCAT & 70 \\
\hline & R-TGCCGTGGGTGGAATCA & \\
\hline \multirow{2}{*}{ KDM1A } & F-CGCAAAGGAAACTATGTAG & 261 \\
\hline & R-ATTATTGAGGACGTTGAAGTC & \\
\hline \multirow[t]{2}{*}{ KDM2A } & F-AGCCTTGACCTCAGTTGGACC & 251 \\
\hline & R-CGGAGCTTGCTGCGATTG & \\
\hline \multirow[t]{2}{*}{ KDM2B } & F-GAGTCAGAGGGCGTGGTC & 202 \\
\hline & R-CACACTCACTCCTCCGCTTGG & \\
\hline \multirow[t]{2}{*}{ KDM3A } & F-AACATGGTGTATTGCGGGTAG & 232 \\
\hline & R-CCTTTGACGGCTCGCTTCC & \\
\hline \multirow[t]{2}{*}{ KDM4A } & F-GGCCATGACTGTGCGAGAG & 312 \\
\hline & R-AAGTCCATGTCTTCGGTGTGC & \\
\hline \multirow[t]{2}{*}{ KDM4B } & F-AATGTGTGTACTGCCGGAAAC & 284 \\
\hline & R-CTGCGTGGTGGTGCCGATGAC & \\
\hline \multirow[t]{2}{*}{ KDM5A } & F-TTCGGGAGACCTATGGCTATG & 282 \\
\hline & R-CTTTAGCTCCTGGCGACAAC & \\
\hline \multirow[t]{2}{*}{ KDM5B } & F-TTCTCCTTGCCCGACGACTG & 242 \\
\hline & R-CTCTTCTCACTGCCGGGTCTC & \\
\hline \multirow[t]{2}{*}{ KDM5C } & F-ACTTCAACATGCCCGTACAC & 300 \\
\hline & R-AGACCATGCCCACATAGAGCC & \\
\hline \multirow[t]{2}{*}{ KDM6A } & F-AGGATGCCATTAAATGCTAC & 336 \\
\hline & R-GGTCATCTACTTGGCTAGAAG & \\
\hline \multirow[t]{2}{*}{ KDM6B } & F-CACGGCGTGGACTACCTG & 201 \\
\hline & R-CTGGTACTGATAGGCGGTGAG & \\
\hline \multirow[t]{2}{*}{ KDM7A } & F-GGAGTTCATCTGTGACCCAG & 305 \\
\hline & R-TCTCAGTTCTTTTAGGGTTTC & \\
\hline \multirow{2}{*}{ PHF2 } & F-AAGCTGGACTCGGCGCTCTAC & 349 \\
\hline & R-TCCCACCAGCCCCGTTCTTC & \\
\hline \multirow[t]{2}{*}{ PHF8 } & F-GCTCCATGGAGTCCTAAAGCC & 270 \\
\hline & R-GGTGAGAAGCTGGGGTGTAG & \\
\hline \multirow[t]{2}{*}{ KDM8 } & F-CGTGCCAATTCCAGACGTGAC & 228 \\
\hline & R-CACTCCTCGTCCGTGTACCTG & \\
\hline \multirow[t]{2}{*}{ JMJD6 } & F-GAGGCTGGTGGCACGTTGTCC & 321 \\
\hline & R-TCTTCCTGCGGTGCATTG & \\
\hline \multirow[t]{2}{*}{ UTY } & F-TCTGCTCCAGGATAGATCCAG & 200 \\
\hline & R-AATGAGGGCAGTATATCTTC & \\
\hline \multirow[t]{2}{*}{ KDM4C } & F-GATTGGCCATATGTCGTGAAC & 239 \\
\hline & R-TCTCGGCTCACAATATCCTC & \\
\hline \multirow[t]{2}{*}{ KDM1D } & F-AGACCAGCAGCTCCCCTACTG & 258 \\
\hline & R-TCCACCTCCTGAACGCATCG & \\
\hline JHDM1C & F-CTTTGGCTGCTGGATCCGACC & 205 \\
\hline & R-CGGCGAACCACACCACTAAG & \\
\hline CYP51A1 & F-TTGAATGCCGAGGAAGTCTAC & 319 \\
\hline & R-CCAAATCCGCATACAGTTGTG & \\
\hline JARID2 & F-CAATCCCAGCCGAATAGTC & 206 \\
\hline & R-TCCTCAACATCCTCCTCGTCC & \\
\hline JMJD1C & F-GGGAGATGTGTGATGCATGTG & 273 \\
\hline & R-TGACAATGGGATTTAATACC & \\
\hline JMJD4 & F-ATGGAGACGTGGTTGTACCTG & 243 \\
\hline & R-CGTCCCAGTACTCGTTGAGC & \\
\hline JMJD7 & F-CCTCCACTTCTACCGGGACTG & 242 \\
\hline & R-GGCCCTCCAGGACGTCCAG & \\
\hline JMJD8 & F-TTGAGCACCGCCAACACCTAC & 293 \\
\hline & R-CGCTTTCGGCCATAGATCAC & \\
\hline
\end{tabular}


Table 3. Composition of in vitro fertilization (IVF) medium.

\begin{tabular}{lc}
\hline Component & For $1000 \mathrm{~mL}$ \\
\hline $\mathrm{NaCl}$ & $6.66 \mathrm{~g}$ \\
$\mathrm{KCl}$ & $0.238 \mathrm{~g}$ \\
$\mathrm{MgCl} \cdot 6 \mathrm{H}_{2} \mathrm{O}$ & $0.1 \mathrm{~g}$ \\
$\mathrm{CaCl}_{2} \cdot 2 \mathrm{H}_{2} \mathrm{O}$ & $0.294 \mathrm{~g}$ \\
$\mathrm{NaH}_{2} \mathrm{PO}_{4} \cdot 2 \mathrm{H}_{2} \mathrm{O}$ & $0.062 \mathrm{~g}$ \\
$\mathrm{NaHCO}_{3}$ & $2.09 \mathrm{~g}$ \\
Sodium pyruvate & $27.5 \mathrm{mg}$ \\
Sodium lactate & $1.121 \mathrm{~g}$ \\
Glucose & $2.5 \mathrm{~g}$ \\
HEPES & $2.4 \mathrm{~g}$ \\
BSA & $6 \mathrm{~g}$ \\
Penicillin & $100 \mathrm{mg}$ \\
Streptomycin & $40 \mathrm{mg}$ \\
\hline
\end{tabular}

Table 4. Composition of in vitro culture (IVC) medium.

\begin{tabular}{lccc}
\hline Chemical & $10 \mathrm{~mL}$ & $100 \mathrm{~mL}$ & $1000 \mathrm{~mL}$ \\
\hline $\mathrm{NaCl}$ & 0.067 & 0.67 & 6.7 \\
$\mathrm{KCl}$ & 0.0023 & 0.0231 & 0.231 \\
$\mathrm{NaHCO}_{3}$ & 0.022 & 0.22 & 2.2 \\
L-glutamine & 0.0015 & 0.015 & 0.15 \\
PSG & $5 \mu \mathrm{L}$ & $50 \mu \mathrm{L}$ & $500 \mu \mathrm{L}$ \\
MEM & $100 \mu \mathrm{L}$ & $1 \mathrm{~mL}$ & $10 \mathrm{~mL}$ \\
BME & $200 \mu \mathrm{L}$ & $2 \mathrm{~mL}$ & $20 \mathrm{~mL}$ \\
Pyruvate stock & $100 \mu \mathrm{L}$ & $1 \mathrm{~mL}$ & $10 \mathrm{~mL}$ \\
L(+)-lactate & 0.0055 & 0.055 & 0.55 \\
BSA & 0.03 & 0.3 & 3.0 \\
\hline
\end{tabular}

\section{Immunofluorescence for bovine early embryos at various stages}

Early embryos at various developmental stages were collected to detect the expression levels of JMJD1C at each stage using immunofluorescence. The embryos were fixed in $4 \%$ paraformaldehyde at room temperature for $30 \mathrm{~min}$. After a PBS wash, they were transferred to PBS with $1 \%$ Triton $\mathrm{X}-100$ and incubated overnight with $\mathrm{CO}_{2}$. The embryos were then washed with PBS, blocked using a 5\% BSA (Sigma) solution for $1 \mathrm{~h}$, and incubated with the anti-JMJD1C primary antibody (ab130922; Abcam, Cambridge, England) at 1:100 dilution in PBS for $1 \mathrm{~h}$ in a $\mathrm{CO}_{2}$ incubator. After a PBS wash, the embryos were incubated for $2 \mathrm{~h}$ in a 1:100 solution of FITC-labeled secondary antibody (Boster, Pleasanton, CA, USA) in PBS in a $\mathrm{CO}_{2}$ incubator. This was followed by treatment with DAPI (Sigma) for $5 \mathrm{~min}$. After washing and mounting, JMJD1C expression was observed under a fluorescence microscope.

\section{Design and synthesis of JMJD1C siRNA}

The JMJD1C siRNAs were formulated by GenePharma (Shanghai, China). The sequences are shown in Table 5.

\section{Oocyte microinjection}

The MII oocytes were cleaned, randomly divided into two groups, and placed in IVC droplets that contained Cytochalasin B (CB). Subsequently, $1 \mu \mathrm{L}$ each of JMJ1C-bos-2149 siRNA 
and JMJD1C-bos-NC siRNA, both at $20 \mu \mathrm{M}$ concentration, were added to the plate. A holding pipette (Beijing Zheng Tian Yi Science and Trade, Beijing, China) with an outer diameter of 100-150 $\mu \mathrm{m}$ was bent to an angle of approximately $30^{\circ}$ to hold the oocytes and a glass capillary tube (GD-1; Narishige, Tokyo, Japan) was used as the injection needle. The needle was used to aspirate the siRNA, pierce the oocyte membrane, and inject the siRNA (10 picoliter) into the cytosol. The arm of the microinjector was adjusted to inject each of the oocytes individually.

\begin{tabular}{ll}
\multicolumn{2}{l}{ Table 5. JMJD1C siRNA sequences. } \\
\hline Interference site & Sequence $\left(5^{\prime}-3^{\prime}\right)$ \\
\hline JMJD1C-bos-2149 & Sense strand: GCACUUAUUGGGUCAGAUATT \\
Negative Control & Antisense strand: UAUCUGACCCAAUAAGUGCTT \\
& Sense strand: UUCUCCGAACGUGUCACGUTT \\
& Antisense strand: ACGUGACACGUUCGGAGAATT \\
\hline
\end{tabular}

\section{Statistical analysis}

SPSS17.0 was used to analyze the data on zygote cleavage and blastocyst rates.

\section{RESULTS}

\section{Gene expression of HDM family members in GV and MII bovine oocytes (JMJD1C screening)}

qRT-PCR was employed to compare the mRNA expression levels of HDM family genes in GV and MII bovine oocytes. We found that the relative expression of JMJD1C was the highest in GV and MII bovine oocytes while KDM1A, KDM1B, KDM2A, KDM3A, KDM4A, KDM5A, KDM5C, $K D M 6 B$, and $K D M 7$ also had considerably higher levels of expression (Figure 1).

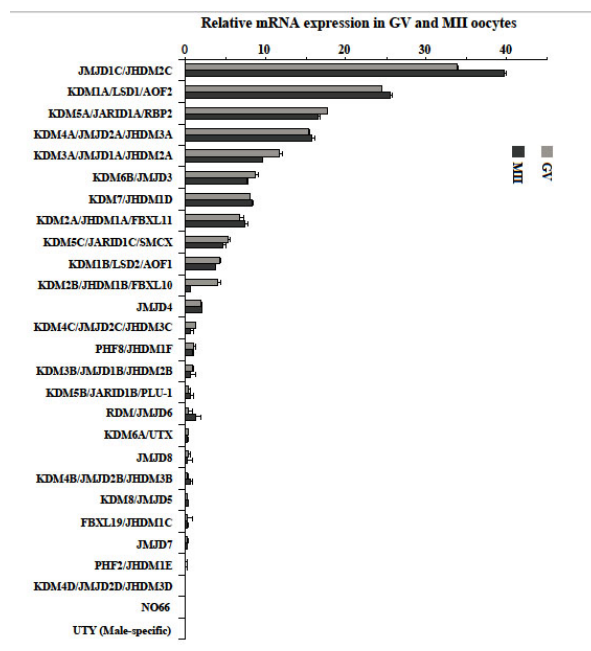

Figure 1. Expression of HDM genes in GV and MII bovine oocytes. Relative mRNA expression levels of HDM genes in GV and in MIl bovine oocytes detected by qRT-PCR. GAPDH gene was used as the internal control. The values are representative of three replicates and indicated as mean \pm standard error. 


\section{JMJD1C expression during early bovine embryonic development}

Since JMJD1C showed the highest relative expression in bovine oocytes, early bovine embryos were collected at different developmental stages after IVF and analyzed for expression and localization of JMJD1C using immunofluorescence. JMJD1C was found to be expressed primarily in the nucleus at all early embryonic stages (Figure 2).

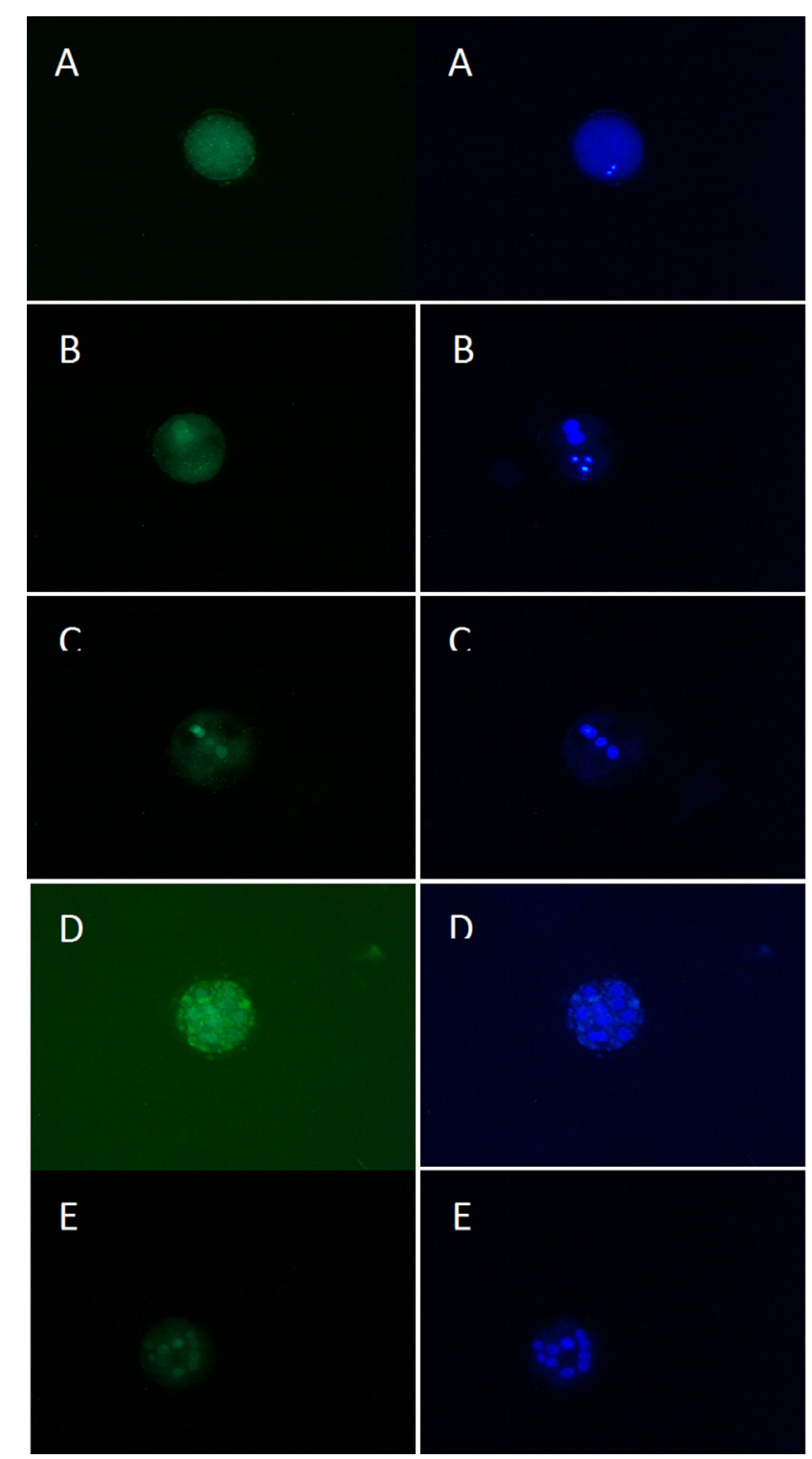

Figure 2. JMJD1C protein localization in early developmental stages of bovine embryos. (A) 2-cell stage, (B) 4-cell stage, (C) 8-cell stage, (D) morula, (E) blastocyst; FITC (left column) represents JMJD1C expression sites and DAPI (right column) represents DNA loci. 


\section{siRNA screening and the effect of JMJD1C silencing in early bovine embryos}

The JMJD1C siRNA knockdown sites in GV oocytes and the fragments amplified by qRTPCR primers are shown in Figure 3A. qRT-PCR detected relatively high levels of expression of JMJD1C mRNA even after interference by different siRNAs. Figure 3B shows that the JMJD1Cbos-2149 siRNA had a JMJD1C knockdown efficiency of approximately $80 \%$ compared to the blank control group $(P<0.01)$ and $60 \%$ compared to the negative control group $(P<0.01)$ (Figure 3B), which prompted its use in subsequent trials in zygotes.

After microinjection of SiRNA, the MIl oocytes were fertilized in vitro and the zygotic cleavage and development of early embryos were observed. The JMJD1C-bos-2149 siRNA-treated group showed a significant reduction in the zygote cleavage rate $(57.1 \pm 0.07 \%)$ and blastocyst rate $(50 \pm 0.01 \%)$ after IVF compared to the control siRNA-treated group. Both the results were statistically significant $(P<0.05)$ (Figure $3 C)$.
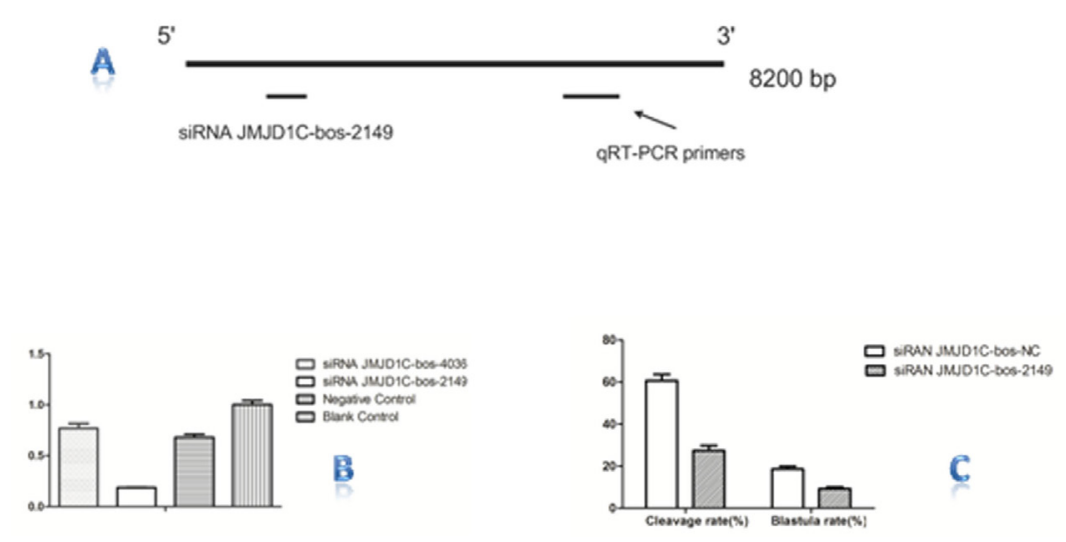

Figure 3. Effect of JMJD1C silencing on zygote cleavage and blastocyst formation in early bovine embryos. (A) siRNA knockdown sites and fragments amplified by qRT-PCR primers. (B) Effects of transfection of JMJD1C siRNAs that targeted different interference sites in bovine oocytes. The knockdown efficiency of the JMJD1C-bos-2149 siRNA was approximately $80 \%$ compared with the blank control group $(P<0.01)$ and $60 \%$ compared with the negative control (NC) group $(P<0.01)$; (C) Effects of siRNA on zygote cleavage rate and blastocyst rate of early bovine embryos $(P<0.05)$.

\section{DISCUSSION}

Our results show that, of all known HDM genes, the mRNA levels of KDM1A, KDM2A, KDM3A, JMJD1C, KDM4A, KDM5A, KDM5C, KDM6B, and KDM7 were high in both GV and in vitro-matured MII bovine oocytes. Canovas et al. have previously reported that the high expression of KDM6B (JMJD3) mRNA in MII oocytes affected the blastocyst rate after the initiation of zygotic cleavage (Canovas et al., 2012). Antony et al. used mouse stem cell lines to stably overexpress the H3K9 trimethylation (H3K9me3)-specific demethylase KDM4B (JMJD2B) and found that it could significantly increase the blastocyst rate of reconstructed embryos after nuclear transfer. However, they did not investigate the underlying molecular mechanisms further (Antony et al., 2013).

Most of the HDM genes analyzed in this study belong to the KDM family. The KDM family of proteins reduces the level of histone lysine methylation and these proteins are also known as histone lysine demethylases. At different sites, lysine has different forms of methylation, which result 
in significant differences in gene regulation. There have been relatively few detailed studies on $\mathrm{H} 3 \mathrm{~K} 9$ methylation, which is an important epigenetic marker for heterochromatin. In the process of cell division, heterochromatin can be inherited stably and plays important roles in genomic stability, centromere function, silencing of repetitive DNA fragments, regulation of gene expression and cell differentiation.

All the JMJD1 family members contain the JMJC domain and are capable of specifically removing $\mathrm{H} 3 \mathrm{~K} 9 \mathrm{me} 1$ and $\mathrm{H} 3 \mathrm{~K} 9 \mathrm{me} 2$ methylation modifications but not H3K9me3 (Okada et al., 2007; Kim et al., 2010; Kim et al., 2012). It is now generally accepted that JMJD1C is the third member of the KDM3 family, i.e., KDM3C. In addition to the common JMJC domain that is shared by the three members, JMJD1C also contains a C2HC4-type zinc finger-like motif and a thyroid hormone receptor $\beta$-binding domain. JMJD1C has unique functions compared to the other two members, KDM3A and KDM3B. Studies have shown that the promoter region of JMJD1C gene contains binding sites for POU5F1/OCT-4, the key transcription factor in stem cells for maintaining pluripotency. Thus, POU5F1/OCT-4-mediated JMJD1C transcription might play an important role in the regulation of gene expression in undifferentiated stem cells (Chen et al., 2013). JMJD1A is a histone $\mathrm{H} 3 \mathrm{~K} 9$ demethylase that can interact with genes associated with embryonic stem cell pluripotency, such as Nanog, Tcl1, Tcfcp2l1, and Zfp57. JMJD1A is an essential regulator in the development of embryonic stem cells (Loh et al., 2007).

Kuroki et al. (2013) found that JMJD1C plays an important role in mouse spermatogenesis using JMJD1C knockout male mice model. They showed gradual reduction in germ cells at 3 months, leading to infertility and testicular fine tubule malformation and failed to produce normal primordial germ cells. JMJD1C is expressed at high levels in mouse spermatogonia and is essential for male germ cell development.

At the 8-cell stage of bovine zygotes, large-scale chromatin remodeling including changes in the level of DNA methylation and post-transcription chemical modification of histone tails occurs in the genomes of male and female pronuclei (Memili and First 1998). This affects the chromatin structure and gene transcriptional activities (Peterson and Laniel 2004) thus promoting zygotic genome activation (Worrad et al., 1995). Histone lysine methylation plays a key regulatory role in the cell differentiation processes and its dynamic changes are related to the transcriptional status of genes (Surani et al., 2007). Such epigenetic elements can specifically inhibit gene expression in embryonic stem cells to maintain pluripotency (Erhardt et al., 2003; Pan et al., 2007). Previous studies with JMJD3 knocked down in bovine oocytes found that JMJD3 completed transcription and translation before the activation of the zygotic genome. Knocking down JMJD3 down-regulated H3K27me3 and significantly decreased the early embryonic blastocyst rate (Canovas et al., 2012).

JMJD1C, KDM3A, and KDM3B are members of the KDM3 demethylase family. JMJD1C was originally identified as the thyroid hormone receptor-binding protein Trip8 (Lee et al., 1995). It also has a splice variant, s-JMJD1C, which is an auxiliary activator of androgen receptors (Wolf et al., 2007). In this study, JMJD1C expression was detected mainly in the nucleus at 2-cell, 4-cell, 8-cell, 16-cell, morula, and blastocyst stages of early bovine embryos after IVF. After knocking down JMJD1C using siRNA, the early embryo cleavage rate and the blastocyst rate were significantly decreased $(P<0.05)$. These results suggest that JMJD1C plays a key role in the normal development of early bovine embryos; however, the exact mechanism is unclear. DNA methylation and histone chemical modifications, particularly $\mathrm{H} 3 \mathrm{~K} 9$ methylation, interact with each other during germ cell production and spatio-temporally regulate the expression of the genome. H3K9 methylation is a sign of inhibition of gene transcription (Seki et al., 2007; Sasaki and Matsui 2008). Studies have shown that in meiosis, H3K9me1 and H3K9me2 show temporary dynamic changes and have different characteristics in male and female germ cells (Tachibana et al., 
2007). Additionally, studies have shown that in JMJD1C knockout male mice, the number of germ cells gradually decreases with age leading to infertility, and that JMJD1C does not affect H3K9 methylation level of the whole genome but only at specific sites and during specific stages (Kuroki et al., 2013). Therefore, our future research focus will be on the specific functions of JMJD1C in early embryonic development. and the effects of microRNAs regulated by JMJD1C on oocytes.

\section{Conflicts of interest}

The authors declare no conflict of interest.

\section{ACKNOWLEDGMENTS}

Research supported by the Earmarked Fund for Modern Agro-industry Technology Research System (\#CARS-38), Key Program for Science and Technology Development of Jilin Province (\#20140204077NY), and Jilin Province Beef Cattle Industrial Technology System.

\section{REFERENCES}

Antony J, Oback F, Chamley LW, Oback B, et al. (2013). Transient JMJD2B-mediated reduction of H3K9me3 levels improves reprogramming of embryonic stem cells into cloned embryos. Mol. Cell. Biol. 33: 974-983.

Canovas S, Cibelli JB and Ross PJ (2012). Jumonji domain-containing protein 3 regulates histone 3 lysine 27 methylation during bovine preimplantation development. PNAS 109: 2400-2405.

Chen J, Liu H, Liu J, Qi J, et al. (2013). H3K9 methylation is a barrier during somatic cell reprogramming into iPSCs. Nat. Genet. 45: 34-42.

Erhardt S, Su I-h, Schneider R, Barton S, et al. (2003). Consequences of the depletion of zygotic and embryonic enhancer of zeste 2 during preimplantation mouse development. Development 130: 4235-4248.

Kim JY, Kim KB, Eom GH, Choe N, et al. (2012). KDM3B is the H3K9 demethylase involved in transcriptional activation of Imo2 in leukemia. Mol. Cell. Biol. 32: 2917-2933.

Kim SM, Kim JY, Choe NW, Cho IH, et al. (2010). Regulation of mouse steroidogenesis by WHISTLE and JMJD1C through histone methylation balance. Nucleic Acids Res. 38: 6389-6403.

Kuroki S, Akiyoshi M, Tokura M, Miyachi H, et al. (2013). JMJD1C, a JmjC domain-containing protein, is required for long-term maintenance of male germ cells in mice. Biol. Reprod. 89: 93.

Lee JW, Choi HS, Gyuris J, Brent R, et al. (1995). Two classes of proteins dependent on either the presence or absence of thyroid hormone for interaction with the thyroid hormone receptor. Mol. Endocrinol. 9: 243-254

Loh YH, Zhang W, Chen X, George J, et al. (2007). Jmjd1a and Jmjd2c histone H3 Lys 9 demethylases regulate self-renewal in embryonic stem cells. Genes Dev. 21: 2545-2557.

Memili E and First NL (1998). Developmental changes in RNA polymerase II in bovine oocytes, early embryos, and effect of a-amanitin on embryo development. Mol. Reprod. Dev. 51: 381-389.

Okada Y, Scott G, Ray MK, Mishina Y, et al. (2007). Histone demethylase JHDM2A is critical for Tnp1 and Prm1 transcription and spermatogenesis. Nature 450: 119-123.

Pan G, Tian S, Nie J, Yang C, et al. (2007). Whole-genome analysis of histone H3 lysine 4 and lysine 27 methylation in human embryonic stem cells. Cell Stem Cell 1: 299-312.

Peterson CL and Laniel MA (2004). Histones and histone modifications. Curr. Biol. 14: R546-R551.

Sasaki H and Matsui Y (2008). Epigenetic events in mammalian germ-cell development: reprogramming and beyond. Nat. Rev. Genet. 9: 129-140.

Seki Y, Yamaji M, Yabuta Y, Sano M, et al. (2007). Cellular dynamics associated with the genome-wide epigenetic reprogramming in migrating primordial germ cells in mice. Development 134: 2627-2638.

Surani M, Hayashi K and Hajkova (2007). Genetic and epigenetic regulators of pluripotency. Cell 128: 747-762.

Tachibana M, Nozaki M, Takeda N and Shinkai Y (2007). Functional dynamics of H3K9 methylation during meiotic prophase progression. EMBO J. 26: 3346-3359.

Wolf SS, Patchev VK and Obendorf M (2007). A novel variant of the putative demethylase gene, s-JMJD1C, is a coactivator of the AR. Arch. Biochem. Biophys. 460: 56-66.

Worrad DM, Turner BM and Schultz RM (1995). Temporally restricted spatial localization of acetylated isoforms of histone H4 and RNA polymerase II in the 2-cell mouse embryo. Development 121: 2949-2959. 